\title{
Vibrotactile masking: Effects of multiple maskers'
}

RICHARD D. GILSON 2

PRINCETON UNIVERSITY

Threshold elevations (TE) were determined at a test site on the left thigh with the simultaneous presentation of combinations of maskers positioned on the trunk. It was found that the resultant TE from groups of up to 10 multiple maskers is nearly equal to the addition of the TE produced by each masker separately.

One of the primary reasons for stimulating multiple loci on the body simultaneously is the large number of different combinations of elements available as patterns for the purpose of cutaneous communication. In theory, the number of pattern combinations possible grows as the nth power of $2, n$ being the number of loci available for stimulation. There have been found, however, some constraints limiting this growth.

One such constraint is that identification of the full complement of elements within a pattern falters rapidly with increases in the number of elements in the pattern, starting with as few as three (Alluisi, Morgan, \& Hawkes, 1965; Geldard \& Hahn, 1961). Geldard and Hahn, employing vibrotactile stimuli, created patterns with the simultaneous stimulation of up to six loci on both of the shoulders, elbows, and wrists in a manner fashioned after the six-dot braille system. Although an alphabetic code was mastered with surprising rapidity, one striking difficulty was encountered when the wrist, elbow, and shoulder of one side were stimulated simultaneously. This resulted in a complete suppression of the sensation at the elbow. An elimination of stimulation, however, at either the wrist or shoulder brought the sensation at the elbow back in full force. A similar difficulty was found by Alluisi et al in a pilot experiment with electrocutaneous stimulation at the same locations. Alluisi et al then proceeded to place stimuli at more widely spread loci in order to avoid the possibility of peripheral neural masking. One electrode was placed on each shoulder blade, one on each arm between the shoulder and the elbow, and one on each side of the trunk just above the belt line. The large increase in the percentage of erroneous responses with increases in the number of loci stimulated was interpreted as evidence of a masking effect, since nearly all of the errors were those of omission. Moreover, because of the nature of the stimulus used (essentially bipolar stimulation) and the wide separation of loci, the results were interpreted as evidence of some central mechanism of masking.

Evidence for a central mechanism of masking has been clearly demonstrated by Gilson (in press), Halliday and Mingay (1961), and Sherrick (1964). Furthermore, while no extensive studies were carried out, indications of an increased suppression or masking with multiple maskers have been reported (Geldard \& Sherrick, 1964; Sherrick \& Gescheider, 1962).

It is the purpose of the present investigation to apply various combinations and numbers of multiple maskers to study their effects more systematically.

\section{APPARATUS}

The instrumentation for signal control and the vibrators used for stimulation have been described previously (Gilson, in press). The only change in the equipment was to separate the masking signal into 10 different channels that controlled the input to the masking vibrators. The output of the $100-\mathrm{W}$ power amplifier was led to a $2-\mathrm{KVA}$ isolation transformer, the output of which in turn was led in parallel to a bank of 10 Superior Electric Type 10 B Variacs. Each of these Variacs independently controlled the voltage delivered to a Sherrick vibrator, up to 10 of which were used as maskers. Each masker could be activated by means of a toggle switch that would close the final circuit to the vibrators. Voltage regulation was such that the effect on the voltage delivered to one vibrator by switching on the other nine vibrators was less than $0.5 \mathrm{~dB}$, and this was considered to be within the error of measurement.

\section{PROCEDURE}

The rationale was to survey a wide range of combinations of maskers in terms of the number employed and the individual effects of maskers on the threshold elevation (TE) at the test site.

The procedure has been described (Gilson, in press) with the one exception that $\mathrm{O}$ initially adjusted all maskers to be used during the experimental session to the standard sensation magnitude. The same Os participated in this study as in the previous one.

The effects of 10 maskers at widespread bodily loci, acting singly, has been reported (Gilson, in press). These data were used to select nine combinations of maskers in the following way: (a) five groups with aggregations of $2,3,5,6$, and 8 maskers were chosen so that their average masking effects (in TE) were the lowest of the 10 maskers examined; (b) three additional groups of 2,3 , and 5 maskers were chosen so that their average masking effects were the highest; (c) one group included all 10 maskers.

\section{RESULTS AND DISCUSSION}

The results are plotted for each $\mathrm{O}$ in Fig. 1. Abscissa values are based on previous data (Gilson, in press) and were determined for each $\mathrm{O}$ as the log sum of the TEs of individual

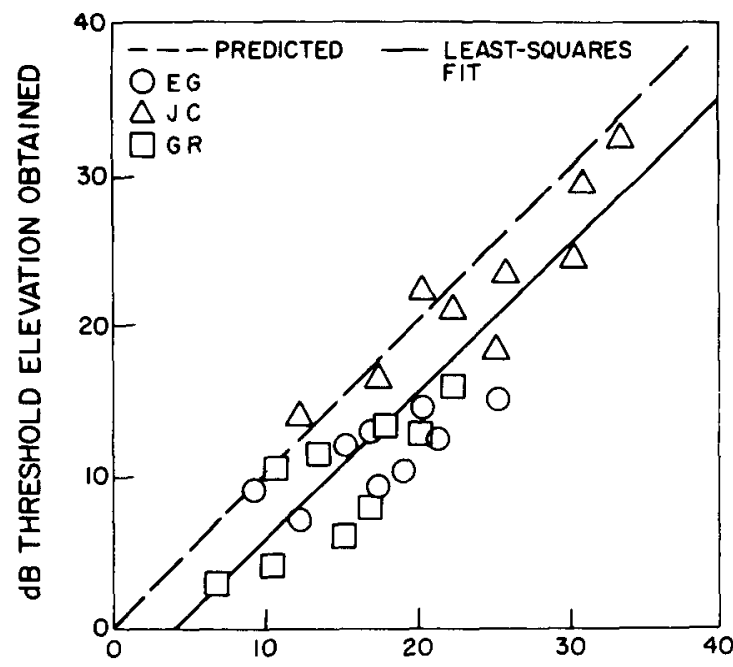

SUMMED THRESHOLD ELEVATION IN dB

Fig. 1. Amount of threshold elevation obtained for groups of multiple maskers as a function of the $\log$ sum of the threshold elevations of individual maskers in each group. 
maskers in each masking group. The log sum was computed by calculating the antilogs for the TEs (in $\mathrm{dB}$ ) of each masker, adding the antilogs, and recalculating the log of that summed value. Ordinate values show the obtained TEs for each $O$ with each masking group. The dashed line shows the predicted values based on perfect summation of the TEs of individual maskers.

It is quite clear that multiple maskers can indeed produce greater TE than a single masker. The highest TE with a single masker was $19.0 \mathrm{~dB}$, while nearly $50 \%$ of the cases tested with multiple maskers resulted in TEs over $19.0 \mathrm{~dB}$, the highest being $32.5 \mathrm{~dB}$. That any combination of multiple maskers produces a higher TE than any single masker, however, does not appear to be the case, since there is a dependency on the choice of individual maskers. This dependency, although somewhat less than perfect in that there appears to be some loss in the addition process, shows the collective TE from a group of maskers to be nearly equal to the addition of individual TEs from the constituents. This conclusion is evident from the least-squares equation which has a slope of nearly $1.0(.95)$ and a small y intercept relative to the scale $(-3.6 \mathrm{~dB})$. As further support that the more intimate relation is with the addition of individual masker effects rather than just sheer number, the Wilcoxon Signed Rank Test shows a significant difference $(p<.01)$ for those groups of maskers chosen as having the same number of maskers but with different predicted summated TE of the individual maskers.

These results are in accord with those patterning studies already cited (Alluisi et al, 1965; Geldard \& Hahn, 1961), which show a general decrement in the identification of the full complement of pattern elements with an increase in the number of elements within a particular pattern. However, one further implication that the present findings make is that it is not merely the number of loci in the patterns that is important, but it is their collective individual effects on a particular locus that is the primary determiner of TE.

\section{REFERENCES}

ALlUISI, E. A., MORGAN, B., \& HAWKES, G. Masking of cutaneous sensations in multiple stimulus presentations. Perceptual \& Motor Skills, 1965, 20, 39-45.

GELDARD, F. A., \& HAHN, J. F. Virginia Cutaneous Project, Progress Report No. 45, 1961.

GELDARD, F. A., \& SHERRICK, C. E. Princeton Cutaneous Research, Project Report No. 4, 1964.

GILSON, R. D. Vibrotactile masking: Some spatial and temporal aspects. Perception \& Psychophysics, in press.

HALLIDAY, A. M., \& MINGAY, R. Retroactive raising of a sensory threshold by a contralateral stimulus. Quarterly Journal of Experimental Psychology, 1961, 13, 1-11.

SHERRICK, C. E. Effects of double simultaneous stimulation of the skin. American Journal of Psychology, 1964, 77, 42-53.

SHERRICK, C. E., \& GESCHEIDER, G. Virginia Cutaneous Project, Progress Report No. 47, 1962.

\section{NOTES}

1. This study was supported by Grant NB-04755 from the National Institutes of Health, U.S. Department of Health, Education and Welfare.

2. Now at Naval Aerospace Medical Institute, Pensacola, Fla. 32512.

(Accepted for publication September 20, 1968.) 